\section{Dr. Varaprasad and Dr. Agrawal reply}

\section{To the Editor:}

We appreciate the opportunity to discuss our case series of posterior reversible encephalopathy syndrome (PRES) in systemic lupus erythematosus (SLE) and comment on the neuroimaging. We agree with Wang, et $a l^{1}$ that $\mathrm{T} 2$ white matter hyperintensities on magnetic resonance imaging (MRI) should be complemented by diffusion-weighted imaging (DWI) and apparent diffusion coefficient (ADC) mapping 2,3 .

In our case series, patients developed PRES in the settings of active SLE: nephritis $(\mathrm{n}=10)$, hypertension $(\mathrm{n}=13)$, those taking oral steroids $(n=7)$, and those taking cyclophosphamide pulses $(n=4 ; 2$ developed PRES within 2 days of infusion). All these are known risk factors for developing PRES. They presented with a symptom complex of seizures, altered sensorium, visual abnormalities, headache, vomiting, and reversible focal deficits $^{4}$. All had characteristic MRI features with bilateral white matter hyperintensities involving not only the classical occipital and parietal lobes but also the frontotemporal and cerebellar areas in 2 cases. DWI and ADC mapping were done to rule out infarcts due to vasculitis (not elaborated in original article). Cerebrospinal fluid analysis and blood cultures were also done to rule out infectious etiology.

The differential diagnosis of PRES in SLE includes CNS infections, seizure disorder, stroke, demyelination, and vasculitis. Reversible periictal imaging changes in patients with isolated seizures are usually confined to the hippocampus or region of epileptic discharge as focal parenchymal T2 hyperintensity and restricted diffusion ${ }^{5}$.

PRES itself can be a manifestation of endothelial dysfunction and in severe cases with extensive lesions, the MRI may show areas of pseudonormalized ADC values, with DWI suggestive of infarcts that affect the prognosis ${ }^{6}$. Apart from the typical MRI features, PRES may also show atypical features such as unilateral lesions, involvement of anterior circulation, cortical lesions, and hemorrhage into lesions ${ }^{2}$.

In practice, the diagnosis of PRES in SLE should be based on characteristic clinical symptoms, with MRI findings being confirmatory.
IRLAPATI RAJENDRA VARAPRASAD, MD, Department of Rheumatology, Nizam's Institute of Medical Sciences, Punjagutta, Hyderabad; SUMEET AGRAWAL, MD, DM, Consultant, Division of Rheumatology and Clinical Immunology, Medanta-The Medicity, Gurgaon, India, PIN 500082. Address correspondence to Dr. Agrawal; E-mail: Sumeet.drsumeetagrawal@gmail.com

\section{REFERENCES}

1. Wang X-K, Zhang H. Is posterior reversible encephalopathy syndrome underestimated in systemic lupus erythematosus? [letter]. J Rheumatol 2011;38:2491.

2. Lee VH, Wijdicks EF, Manno EM, Rabinstein AA. Clinical spectrum of reversible posterior leukoencephalopathy syndrome. Arch Neurol 2008;65:205-10.

3. Bartynski WS. Posterior reversible encephalopathy syndrome, part 2: controversies surrounding pathophysiology of vasogenic edema. AJNR Am J Neuroradiol 2008;29:1043-9.

4. Varaprasad IR, Agrawal S, Prabu VN, Rajasekhar L, Kanikannan MA, Narsimulu G. Posterior reversible encephalopathy syndrome in systemic lupus erythematosus. J Rheumatol 2011;38:1607-11.

5. Kim JA, Chung JI, Yoon PH, Kim DI, Chung TS, Kim EJ, et al. Transient MR signal changes in patients with generalized tonicoclonic seizure or status epilepticus: periictal diffusion-weighted imaging. AJNR Am J Neuroradiol 2001;22:1149-60.

6. Covarrubias DJ, Luetmer PH, Campeau NG. Posterior reversible encephalopathy syndrome: prognostic utility of quantitative diffusion-weighted MR images. AJNR Am J Neuroradiol 2002;23:1038-48.

J Rheumatol 2011;38:11; doi:10.3899/jrheum.110821 